\title{
Vapor-solid-solid grown Ge nanowires at integrated circuit compatible temperature by molecular beam epitaxy
}

\author{
Zhongyunshen Zhu, ${ }^{1,2,3, a)}$ Yuxin Song, ${ }^{1, a), b)}$ Zhenpu Zhang, ${ }^{1,2}$ Hao Sun, ${ }^{1}$ Yi Han, ${ }^{1,3}$ \\ Yaoyao Li, ${ }^{1}$ Liyao Zhang, ${ }^{1}$ Zhongying Xue, ${ }^{1}$ Zengfeng $\mathrm{Di},{ }^{1}$ and Shumin Wang ${ }^{1,2,4, \mathrm{~b})}$ \\ ${ }^{1}$ State Key Laboratory of Functional Materials for Informatics, Shanghai Institute of Microsystem \\ and Information Technology, Chinese Academy of Sciences, Shanghai 200050, China \\ ${ }^{2}$ School of Physical Science and Technology, ShanghaiTech University, Shanghai 201210, China \\ ${ }^{3}$ University of Chinese Academy of Sciences, Beijing 100190, China \\ ${ }^{4}$ Department of Microtechnology and Nanoscience, Chalmers University of Technology, Gothenburg 41296, \\ Sweden
}

(Received 16 June 2017; accepted 20 August 2017; published online 5 September 2017)

\begin{abstract}
We demonstrate Au-assisted vapor-solid-solid (VSS) growth of Ge nanowires (NWs) by molecular beam epitaxy at the substrate temperature of $\sim 180^{\circ} \mathrm{C}$, which is compatible with the temperature window for Si-based integrated circuit. Low temperature grown Ge NWs hold a smaller size, similar uniformity, and better fit with Au tips in diameter, in contrast to Ge NWs grown at around or above the eutectic temperature of Au-Ge alloy in the vapor-liquid-solid (VLS) growth. Six $\langle 110\rangle$ growth orientations were observed on Ge (110) by the VSS growth at $\sim 180^{\circ} \mathrm{C}$, differing from only one vertical growth direction of Ge NWs by the VLS growth at a high temperature. The evolution of NWs dimension and morphology from the VLS growth to the VSS growth is qualitatively explained by analyzing the mechanism of the two growth modes. Published by AIP Publishing.

[http://dx.doi.org/10.1063/1.4990602]
\end{abstract}

\section{INTRODUCTION}

Ge nanowires (NWs) have promising electronic properties $^{1,2}$ and play an increasingly important role in various applications, such as high speed transistors, ${ }^{3-5}$ photodetectors, ${ }^{6,7}$ and the anode material of high capacity lithium-ion batteries. ${ }^{8}$ Conventional Ge NW growth via the Au-assisted vapor-liquid-solid (VLS) process takes place above the AuGe eutectic temperature $\left(T_{\mathrm{e}} \approx 361^{\circ} \mathrm{C}\right) .{ }^{9-11}$ However, for the VLS growth of NWs, it is difficult to control the NW size and position due to surface migration and coalescence of the catalysts during the growth. Furthermore, NW tapering or kinking is more likely to occur in the VLS growth, thus affecting the shape of NWs. ${ }^{12}$ These characteristics of VLS oppose the requirements for the practical application of bottom-up NWs in large-scale integrated circuits (ICs), which require the selective growth of NWs in ordered patterns. Because of this, the vapor-solid-solid (VSS) growth of NWs is worth taking into practice to overcome the drawback of VLS, since both the size and position of the solid catalysts can be determined due to the solid phase of the catalysts, thus minimizing catalyst migration and coalescence. ${ }^{13,14}$

To date, a few reports covered the VSS growth of Ge NWs, in the catalyst-Ge systems as follows: Ni-Ge [at $300-375^{\circ} \mathrm{C}$ via chemical vapor deposition (CVD) $],{ }^{15} \mathrm{Mn}-\mathrm{Ge}$ (at $350{ }^{\circ} \mathrm{C}$ via CVD), ${ }^{16} \mathrm{Cu}-\mathrm{Ge}$ (at $200-300^{\circ} \mathrm{C}$ via $\mathrm{CVD}$ ), ${ }^{14}$ and $\mathrm{Au}-\mathrm{Ge}$ [at $255-330^{\circ} \mathrm{C}$ via $\mathrm{CVD}$ or physical vapor deposition (PVD)]. ${ }^{17,18}$ To meet the temperature compatibility of current ICs when the Ge NWs are required to be grown on

\footnotetext{
a) Zhu and Y. Song contributed equally to this work.

b) Authors to whom correspondence should be addressed: songyuxin@mail. sim.ac.cn and shumin@mail.sim.ac.cn.
}

processed circuits, ${ }^{19}$ a low synthetic temperature of Ge NWs should be utilized. The growth with a catalyst such as $\mathrm{Ni}$ or Mn will occur above $300^{\circ} \mathrm{C}$, which can considerably damage the processed circuit. Although $\mathrm{Cu}$ and $\mathrm{Au}$ are possible alternative catalysts for Ge NW growth after IC processing, compared to $\mathrm{Au}, \mathrm{Cu}$ is chemically more active and immature in the deposition process. On the other hand, as an established steady catalyst, Au exhibits a significant position in NW growth. Although the VSS growth of Au-assisted Ge NWs has been achieved at a temperature lower than $T_{\mathrm{e}}$ by utilizing ultra-high vacuum (UHV) $\mathrm{CVD}^{17}$ or $\mathrm{PVD}^{18}{ }^{18}$ neither of these reports produced a detailed analysis of VSS grown Ge NWs. Furthermore, the growth temperature ranged from 255 to $330^{\circ} \mathrm{C}$, which is difficult to deal with present IC technique. In addition, in contrast to the CVD, molecular beam epitaxy (MBE) is a far-from-equilibrium physical process, ${ }^{20}$ which commonly requires much lower growth temperature than the chemical reactions in CVD, and can achieve higher material purity and lower material consumption. $^{21}$

Here, we demonstrate Au catalyzed Ge NWs grown on Ge (110) substrates through the VSS process by MBE. The growth temperature is lowered to $220^{\circ} \mathrm{C}$ (the thermocouple temperature, corresponding actual temperature is $\sim 180^{\circ} \mathrm{C}$ ), which is much lower than $T_{\mathrm{e}}$ even if the Gibbs-Thomson effect would be considered ${ }^{22}$ and well satisfies the compatibility with the temperature window for Si-based IC. Meanwhile, for group-IV photonics, such a low growth temperature of Ge NWs can promote the possibility to incorporate Sn, while GeSn NW potentially becomes a direct bandgap material for high efficiency light emission. ${ }^{23}$ For comparison, we also grew Ge NWs at above $T_{\mathrm{e}}$ as reference. Our result shows that the diameter as well as the density of Ge NWs decreases with 
decreasing growth temperature from above $T_{\mathrm{e}}$ to below $T_{\mathrm{e}}$, while the size uniformity does not change. Furthermore, the VSS process leads to NWs in three $\langle 110\rangle$ growth directions simultaneously: vertical, lateral, and $30^{\circ}$-tilted to surface, in contrast to only one vertical direction in the VLS growth. Besides, the diameter of the Au particles approximately fits that of the NWs in the VSS process, but becomes much smaller during the VLS growth. All these morphological differences in both the growth orientation and the NW diameter can be explained by the fundamental growth mechanism in the VSS and the VLS processes. The Ge (110) substrate is selected because Ge NWs grow along $\langle 110\rangle$ by VLS without atomic hydrogen environment, ${ }^{24}$ and most of the Ge NWs are vertical to the $\mathrm{Ge}(110)$ substrate. $^{10}$

\section{EXPERIMENT}

Ge NWs were grown via a DCA P450 solid source MBE system. Prior to the growth, Ge substrates were degassed at $400^{\circ} \mathrm{C}$ for $30 \mathrm{~min}$ and further heated to $650^{\circ} \mathrm{C}$ for an hour to remove native oxides. Then, $\sim 2 \mathrm{~nm}$ thick Au film was deposited onto cleaned Ge substrates at room temperature via electron beam evaporation and annealed at $450{ }^{\circ} \mathrm{C}$ for $30 \mathrm{~min}$ to form nanoparticles. Finally, Ge NWs were grown on Ge (110) substrates under different growth temperatures, but a fixed Ge growth rate of $\sim 0.010 \mathrm{~nm} / \mathrm{s}$ and total growth time of $3.5 \mathrm{~h}$ under UHV of $\sim 1.0 \times 10^{-9}$ Torr. At this low growth rate, the VSS process is responsible for the Ge NW growth at a temperature far below $T_{\mathrm{e}} \cdot{ }^{17}$ Due to the distinction in growth mechanism for Ge NWs grown above and below $T_{\mathrm{e}}$, we chose growth temperatures of $220^{\circ} \mathrm{C}, 290^{\circ} \mathrm{C}, 380^{\circ} \mathrm{C}$, and $470^{\circ} \mathrm{C}$ to exhibit the morphologic transition. Moreover, two-step growth from $470{ }^{\circ} \mathrm{C}$ to $220^{\circ} \mathrm{C}$ (growing at $470^{\circ} \mathrm{C}$ for $1.5 \mathrm{~h}$ then cooling down to $220^{\circ} \mathrm{C}$ to grow for $2 \mathrm{~h}$ ) was experimentally conducted to study the transition from VLS to VSS process. Postgrowth characterization for morphology of Ge NWs employed scanning electron microscope (SEM) at different angles and transmission electron microscope (TEM) to examine the crystalline structure.

Here, all temperatures expressed were read via a thermocouple behind the substrate heater. In fact, the actual substrate temperature is about $40^{\circ} \mathrm{C}$ below the thermocouple-measured data in the range of $200-300^{\circ} \mathrm{C}$ calibrated by the melting point of $\mathrm{Sn}$. The method is carried out as followed. First, the in-situ reflection high-energy electron diffraction (RHEED) pattern of the oxide-removed Ge (001) substrate was observed at the thermocouple-measured temperature of $80^{\circ} \mathrm{C}$. Then, $1 \mathrm{~nm} \mathrm{Sn}$ was deposited onto the Ge surface and the RHEED pattern disappeared. Finally, the temperature slowly increased to $270^{\circ} \mathrm{C}$, until the RHEED pattern of Ge (001) reappeared, when the solid Sn melted and coalesced, partially exposing the Ge surface. We propose that the read temperature at the change point corresponded to the melting point of Sn. Under atmospheric pressure, the melting point of Sn was $231.9^{\circ} \mathrm{C}$. Two points should be noted here for the validity of this method. First, the solubility of $\mathrm{Sn}$ in $\mathrm{Ge}$ is low (below 1\%) and the eutectic temperature was $\sim 232^{\circ} \mathrm{C}$, resulting in little change to the melting point even when they alloyed. Second, for $\mathrm{Sn}$ in the ultra-high vacuum $\left(\sim 1.0 \times 10^{-9}\right.$ Torr $)$, the melting point only decreased slightly from that under atmospheric pressure, for about $2.8-4.3^{\circ} \mathrm{C} .{ }^{25}$ Both points show that the melting point of $\mathrm{Sn}$ in MBE differed only slightly from that in air. Since the calibration of the true substrate temperature could be credible only in the temperature range close to the Sn melting point, and the precision was not sufficiently high, the thermocouplemeasured temperatures were still used for the comparability of the growth temperatures in our experiment.

\section{RESULTS AND DISCUSSION}

Ge NWs on Ge (110) substrates were successfully grown at $220^{\circ} \mathrm{C}$ by VSS MBE with Au catalyst. Figures 1(a) and 1(b) show the top-view SEM images of as-deposited Au droplets and Ge NWs grown at $220^{\circ} \mathrm{C}$, respectively. In contrast to the high density of Au droplets on the substrate, it shows a relatively lower density of Au-catalyzed NWs. Only less than $50 \%$ of the Au droplets successfully catalyzed NW growth. Figure 1(b) suggests that the large Au nanoparticles are more likely to participate in the NW growth, while the small nanoparticles in Fig. 1(a) may be covered by the 2-dimensional Ge layer, thus remaining hidden in Fig. 1(b). Additionally, some of the hexagonal dots represent the vertical NWs due to the top view shown in Fig. 1(b).

The TEM analysis of a NW grown at $220^{\circ} \mathrm{C}$ is presented in Fig. 2. In Fig. 2(a), the vertical Ge NW was found to be partially buried by a Ge film, which was grown without catalysis, and the boundary between the solid Au catalyst and the Ge NW can be clearly seen. Furthermore, the high resolution TEM (HRTEM) from Fig. 2(b) shows a different lattice configuration between the NW and the catalyst. Moreover, Fig. 2(c) exhibits a HRTEM image of the NW, showing single crystalline property and growth along the

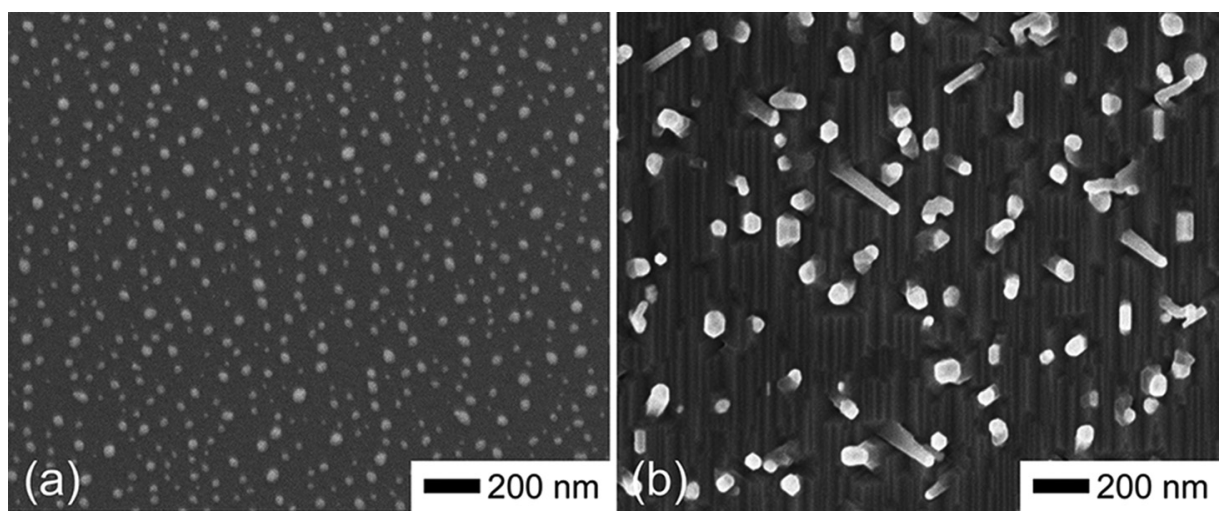

FIG. 1. SEM images of (a) Au droplets on Ge (110) and (b) Ge NWs grown at $220^{\circ} \mathrm{C}$ on $\mathrm{Ge}(110)$ substrate. 

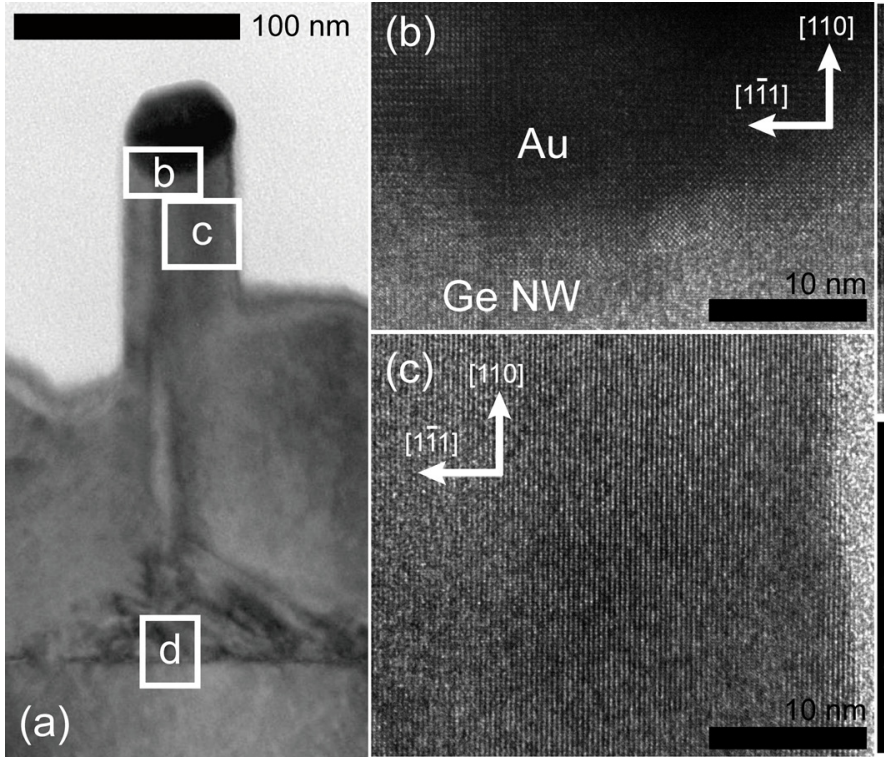

[110] direction, verified by the diffraction pattern in Fig. 2(e). The epilayer/substrate interface can be apparently observed in both Figs. 2(a) and 2(d). Due to an unintentional slight inclination when preparing the specimen via focused ion beam, part of the Ge film lies in front of the bottom of the NW. Therefore, the lattice seen in Fig. 2(d) comprises both the Ge NW and the film. Both are found to be epitaxially grown from the crystal lattice of the substrate. A few edge dislocations [one is marked in Fig. 2(d)] can be found in the interface both within the NW region and in the thin film. For those within the NW region, it remains unclear whether they originate in the NW or the in film. Above the substrate/NW interface, no dislocation was found, showing high crystalline quality.

In comparison, we have investigated Ge NW growth at the selected temperatures on Ge (110) substrate shown in Figs. 3 and 4. Figure 3(a) shows the histogram of Ge NW diameter distribution at the growth temperatures of $220^{\circ} \mathrm{C}$ and $380^{\circ} \mathrm{C}$. The NWs grown at $220^{\circ} \mathrm{C}$ reveal a symmetric and narrow size distribution centered at 40-60 nm in diameter, while the NWs grown at $380^{\circ} \mathrm{C}$ have an asymmetric

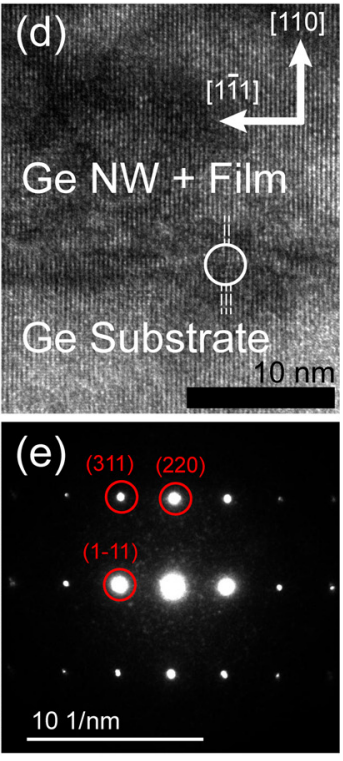

FIG. 2. (a) Low resolution TEM image of the $220^{\circ} \mathrm{C}$ grown sample; (b)-(d) HRTEM images of the positions marked by rectangles in (a); (e) The diffraction pattern from the Ge NW. The white circle and the dash lines in (d) indicate the edge dislocation.

and broad size distribution with the weight on 100-120 nm in diameter. Moreover, to simply compare the size difference between the VSS and VLS growth, the statistical data of vertical NW radius and length, as well as the corresponding fitting curves, are plotted in Fig. 3(b). Here, the modified mass transport model by Johansson was used to fit the data, since our case matches the four assumptions required by this model. ${ }^{26}$ Only vertical NWs were employed for ease of fitting since the source atoms only originated from a direction almost perpendicular to the substrate surface, thanks to the high vacuum in MBE, and therefore, direct deposition of Ge on the NW sidewalls can be ignored. Furthermore, at $220^{\circ} \mathrm{C}$, the solubility of Ge in $\mathrm{Au}$ is quite low because of the solid phase, resulting in the ability to reasonably neglect the direct impingement of source atoms into the catalyst for NW growth. Thus, the expression of the NW growth rate $(\mathrm{d} L / \mathrm{d} t)$ at $220^{\circ} \mathrm{C}$ and $380^{\circ} \mathrm{C}$ can be given by,

$$
\frac{\mathrm{d} L}{\mathrm{~d} t}=-\frac{2 \Omega J_{\mathrm{s}}}{r \cosh \left(L / \lambda_{\mathrm{w}}\right)}
$$
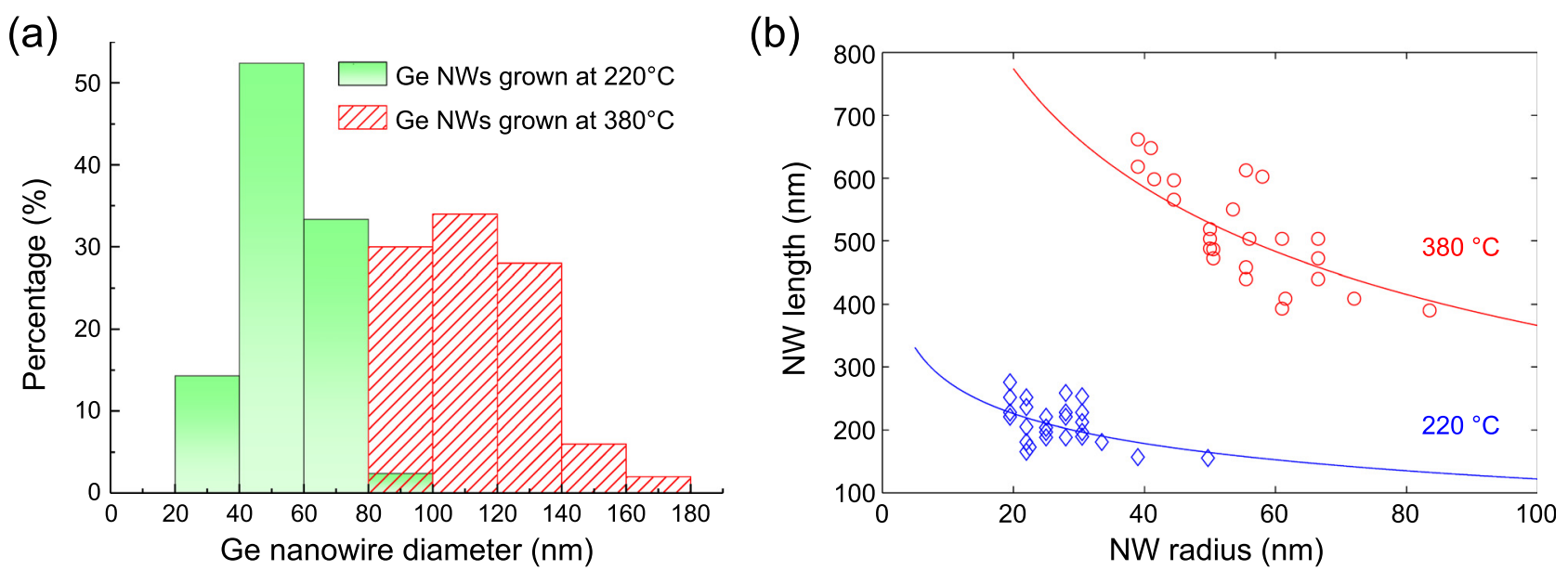

FIG. 3. (a) The diameter histogram of Ge NWs grown at $220^{\circ} \mathrm{C}$ (green bars) and at $380^{\circ} \mathrm{C}$ (red pattern bars); (b) NW radius vs. length data obtained at $220^{\circ} \mathrm{C}$ (blue diamonds) and $380^{\circ} \mathrm{C}$ (red circles) of the vertical NWs. The solid curves are fitting results from the mass transport model in the NW growth. 


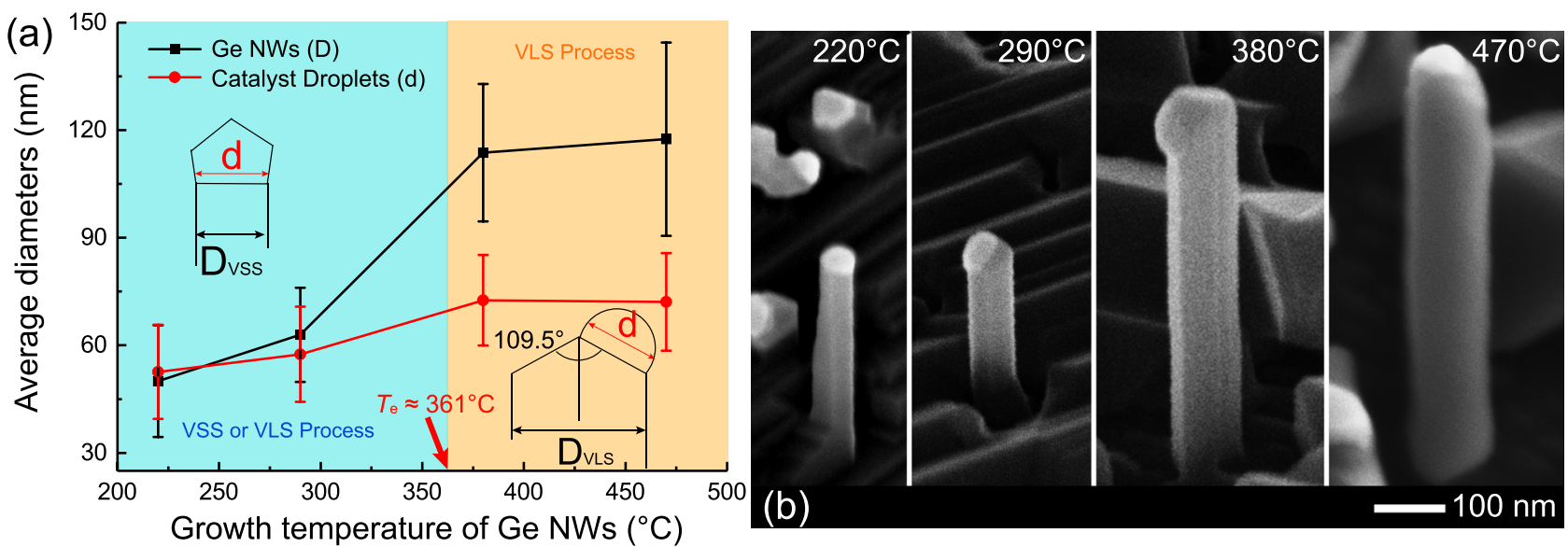

FIG. 4. (a) The histogram of Ge NWs grown at $220^{\circ} \mathrm{C}$ (green bars) and at $470{ }^{\circ} \mathrm{C}$ (red pattern bars). (b) Average diameter and its error bars of Ge NWs (black squares) and the corresponding Au tips (red dots) as a function of growth temperature. (c) Tilted SEM images of single Ge NW grown at different temperatures on $\mathrm{Ge}(110)$.

$$
\frac{\mathrm{d} L}{\mathrm{~d} t}=-\frac{2 \Omega J_{\mathrm{s}}}{r \cosh \left(L / \lambda_{\mathrm{w}}\right)}+2 \Omega \mathrm{R}_{\mathrm{top}}
$$

with $J_{\mathrm{s}}=-\mathrm{R}_{\mathrm{s}} \lambda_{\mathrm{s}} \mathrm{K}_{1}\left(r / \lambda_{\mathrm{s}}\right) / \mathrm{K}_{0}\left(r / \lambda_{\mathrm{s}}\right)$ representing the expression of the adatom flux from the surface to the $\mathrm{NW}\left(\mathrm{K}_{0}\right.$ and $\mathrm{K}_{1}$ are modified Bessel functions of the second kind), where $r$ represents the radius of NWs; $\lambda_{\mathrm{w}}$ and $\lambda_{\mathrm{s}}$ represent the diffusion length of the adatoms on the NW sidewalls and the substrate surface, respectively; $\Omega=2.26 \times 10^{-29} \mathrm{~m}^{-3}$ represents the atom density of $\mathrm{Ge}$; and $\mathrm{R}_{\text {top }}$ and $\mathrm{R}_{\mathrm{s}}$ denote the effective rate of $\mathrm{Ge}$ atoms dissolved in the catalyst and the net deposition on the substrate, respectively. The diffusion lengths of the substrate and NW sides can be roughly fitted from the equations, as $\lambda_{\mathrm{w}}=48 \mathrm{~nm}, \lambda_{\mathrm{s}}=70 \mathrm{~nm}$ at $220^{\circ} \mathrm{C}$ and $\lambda_{\mathrm{w}}=183 \mathrm{~nm}, \lambda_{\mathrm{s}}$ $=124 \mathrm{~nm}$ at $380^{\circ} \mathrm{C}$. The result shows that both diffusion lengths decrease significantly with the growth temperature. Therefore, the NW length and the radius in the VSS growth are much smaller than those in the VLS growth.

Another distinguished change between VSS and VLS is the diameter match of the catalyst and the NW. The average diameter values of not only the Ge NWs but also the catalyst droplets are calculated by counting more than $30 \mathrm{NWs}$ per sample and shown in Fig. 4(a). The average diameter of Ge NWs increases remarkably with growth temperatures from $290^{\circ} \mathrm{C}$ to $380{ }^{\circ} \mathrm{C}$, but otherwise changes slightly. The diameter values of Ge NWs during the VLS process are about twice of that during the VSS process. The corresponding standard deviation also tends to increase with increasing growth temperatures above $T_{\mathrm{e}}$; however, it remains nearly a constant if normalized by the mean values. This indicates that the uniformity of Ge NWs was approximately invariant at each growth temperature. Additionally, the similar trend in the diameters of catalyst droplets with varying growth temperature can be seen in Fig. 4(a). Interestingly, the catalysts had roughly the same diameters as NWs below $T_{\mathrm{e}}$, while the diameters of droplets were much smaller than those of NWs above $T_{\mathrm{e}}$. These statistical data fit our assumption well that the droplets stay only on one of the top $\{111\}$ facets in the VLS process, as the schematics show in the insets of Fig. 3(a), similar to the result in Ref. 10. The droplet diameter $d$ and the NW diameter $D_{\mathrm{VLS}}$ follow the relationship of $D_{\mathrm{VLS}} \approx 2 d \sin \left(109.5^{\circ} / 2\right)$.

Figure 4(b) shows SEM images including a single Ge NW grown at designed growth temperatures on Ge (110). For the sidewall facets, VLS grown NWs can be prisms bounded by four $\{111\}$ surfaces; however, sometimes these had a hexagonal cross-section exposed by four $\{111\}$ in addition to two $\{001\}$ sidewalls $^{18}$ as the NW grown at $380^{\circ} \mathrm{C}$ in Fig. 4(b), while the VSS grown NWs always possess the hexagonal cross-section. For the catalyst/NW interface, as previously indicated from our observation, there are two $\{111\}$ planes inclined $109.5^{\circ}$ in the VLS growth ${ }^{10,18}$ while only one $\{111\}$ plane formed the interface in the VSS growth. Both the NW diameter and length increase when the growth mode transited from VSS to VLS, which is consistent with the result in Figs. 3(b) and 4(a). This dramatic change in volume is primarily determined by the temperature dependent diffusion length of Ge atoms on the substrate and NW sides during MBE growth from the previous estimations and publications. ${ }^{11,27}$ It was reported that $\sim 430{ }^{\circ} \mathrm{C}$ is the optimal temperature for Ge NW growth by VLS. ${ }^{10,11}$ Thus, the Ge diffusion length is supposed to reach a maximum at this growth temperature. Higher temperatures may restrain $\mathrm{Ge}$ diffusing towards Au droplets, but assist Ge to grow on the area between NWs. From this point of view, we can explain that the size of Ge NWs becomes similar at the growth temperatures of $380^{\circ} \mathrm{C}$ and $470{ }^{\circ} \mathrm{C}$ in Fig. 4(b). The diffusion length is further limited by the ultra-low growth temperature far below $T_{\mathrm{e}}$. As a result, less $\mathrm{Ge}$ is capable of travelling from the impingent plane or sidewalls of NWs to the interface between $\mathrm{Au}$ catalyst and Ge to form NWs, causing a small size of NWs. In addition, the interface of the solid Au nanoparticle and the NW seems to be tilted at the growth temperature of $220^{\circ} \mathrm{C}$ and $290^{\circ} \mathrm{C}$. This may suggest that $\mathrm{Ge}$ NWs have one dominant $\{111\}$ top facet in the VSS process.

Furthermore, we compare the growth orientation of Ge NWs at temperatures below and above $T_{\mathrm{e}}$ shown in Fig. 5. Three dominant orientations of Ge NW growth of VSS are observed, delineated in Figs. 5(a) and 5(b) (we assume that [110] is perpendicular to the Ge (110) substrate). The solid 

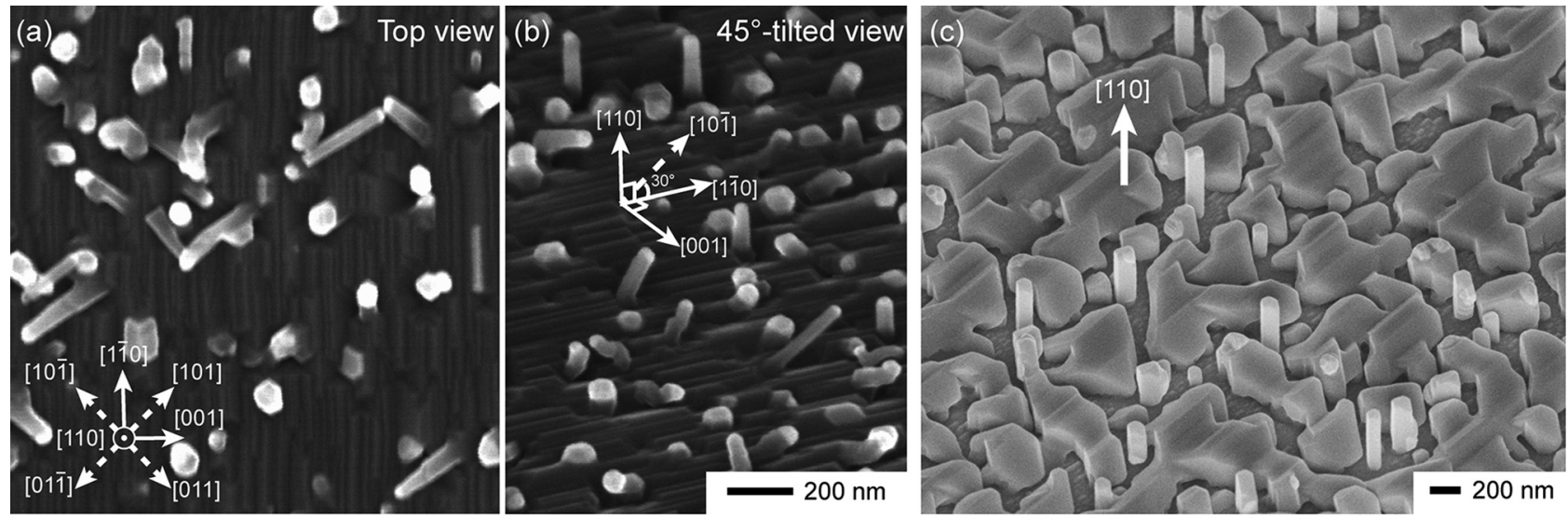

FIG. 5. SEM analysis of Ge NW growth orientation on Ge (110) at (a) $220^{\circ} \mathrm{C}$ (top-view), (b) $220^{\circ} \mathrm{C}\left(45^{\circ}\right.$-tilted view), and (c) $470^{\circ} \mathrm{C}\left(45^{\circ}\right.$-tilted view).

arrow lines denote axes on Ge (110), while the dashed arrow lines are $30^{\circ}$-tilted $\langle 110\rangle$ directions (four equivalent directions including [10-1], [101], [01-1], and [011]). At a growth temperatures above $T_{\mathrm{e}}$, most of Ge NWs prefer to grow along the [110] and other four equivalent directions: [011], [01-1], [101], and [10-1] are rarely observed as illustrated in Fig. 5(c), in agreement with the result of Schmidtbauer et al. ${ }^{10}$ Interestingly, at a low growth temperature, lateral NWs appear along the [1-10] direction. Scrutinizing through different positions of the NW sample grown at $220^{\circ} \mathrm{C}$, we found that the NWs with $30^{\circ}$ inclination all possess a long length, thin diameter, and small Au tips, both shown in Figs. 1(b) and 5(a). This result indicates that the small $\mathrm{Au}$ nanoparticle tends to induce the nucleation occurring along $30^{\circ}$-tilted $\langle 110\rangle$ directions instead of the vertical direction. The possible reason of the larger length of the inclined NWs is that, compared to the vertical directions, additional Ge atoms can directly be deposited onto the NW sidewalls facing the Ge source, leading to more efficient supply of Ge adatoms reaching the catalyst/NW interface to incorporate into the NWs. In Fig. 5(c), we notice that some of NWs have a droplet on the their top or sidewall, while some NWs have lost this possibly because of catalyst spreading $^{24}$ and Ostwald ripening process ${ }^{28}$ during the long growth time of VLS at relatively high temperature. Figure 5(b) also shows the low density of Ge NWs grown at $470{ }^{\circ} \mathrm{C}$ caused by two possible aspects: (i) the catalyst separation is much larger than the surface diffusion length so that some of the adatoms cannot reach the catalyst to form NWs and nucleate as islands, and (ii) long-time and high-temperature growth leads to catalyst migration, which can stop NW growth. Thus, the later stage of growth may be dominated by island growth.

To investigate the transition from the VLS growth to the VSS growth, a sample with two-step growth was prepared. We first grew Ge NWs on Ge (110) at $470{ }^{\circ} \mathrm{C}$ for $1.5 \mathrm{~h}$, followed by the growth at $220^{\circ} \mathrm{C}$ (cooling down to $220^{\circ} \mathrm{C}$ directly) for $2 \mathrm{~h}$, keeping the same total growth time as for the previous samples. As expected, the second step of growth at $220^{\circ} \mathrm{C}$ displays identical three main growth directions, which are [110], [10-1], and [1-10] in Figs. 6(a), 6(b), and 6(c), respectively, similar to the results shown in Figs. 5(a) and 5(b). Moreover, almost every NW grows along the [110] in the first step due to the VLS growth, the same as the result of the $\mathrm{NW}$ growth at $470{ }^{\circ} \mathrm{C}$. This match of orientation between the two-step growth and the direct growth at $220^{\circ} \mathrm{C}$ or $470^{\circ} \mathrm{C}$ suggests that the VSS process occurs when Ge NWs are grown at $220^{\circ} \mathrm{C}$ and the VLS process occurs at $470^{\circ} \mathrm{C}$. It is worth noting that the NW diameters in the first step approximately double those in the second step as shown in Fig. 6, which is consistent with the statistical consequence in Fig. 4(a).

From the standpoint of elementary processes in the NW growth model by Tersoff, ${ }^{29}$ we use a $2 \mathrm{D}$ model shown in Fig. 7(a) to simplify the explanation of the VLS process by MBE. The system is stable if the contact angle $\left(\theta_{c}\right)$ of the droplet situates inside the range marked by two dash lines. Additionally, sidewalls of the NW are $\{111\}$ facets, which have minimum surface energy. Initially, an $\mathrm{Au}$ droplet lies on the surface to catalyze growing a Ge pedestal exposed by $\{111\}$ facets on the Ge (110) substrate illustrated in Fig. 7(b). As the system evolves, the interface becomes so large that $\theta_{c}$ decreases outside the stable range to form a step marked by the blue circle of Fig. 7(c) and then the interface will tend to diminish to a small size and $\theta_{c}$ increases, as shown in Fig. 7(c). When the $\theta_{c}$ falls outside the stable range again, the droplet will depin from the right trijunction, indicated by the arrow in Fig. 7(c). The reason that vertical NWs

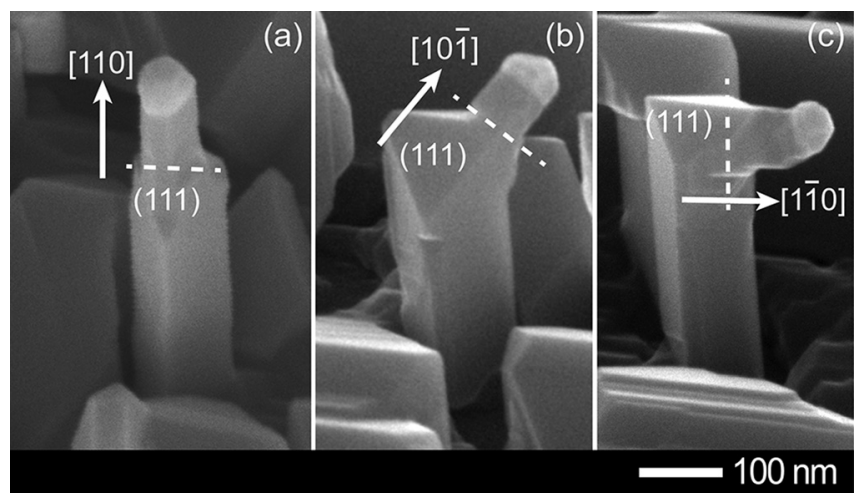

FIG. 6. SEM images of Ge NWs with the two-step growth on Ge (110). The second growth mode is separated from the first one by dash lines and shows three orientations: (a) vertical, (b) $30^{\circ}$-tilted to the surface, and (c) in-plane. 


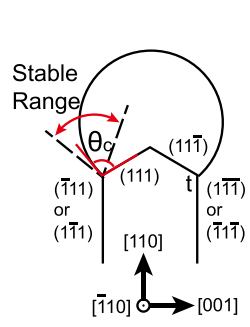

(a)

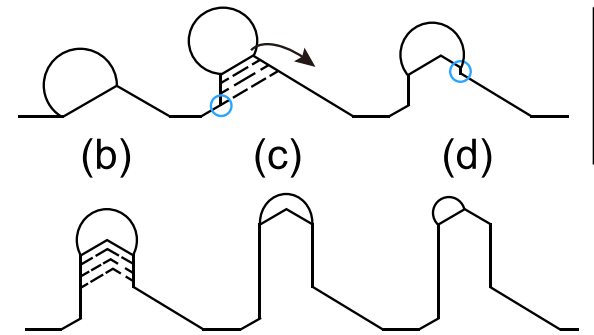

(e) (f) (g)

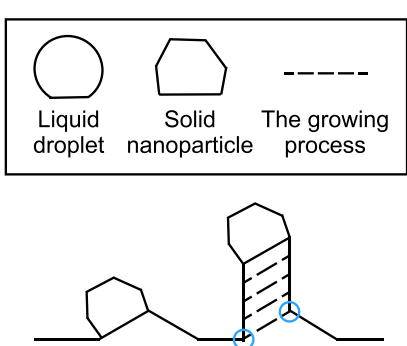

(h)

(i)

FIG. 7. Illustration of growth mechanism of the VLS and the VSS growth. (a) 2D model from the view illustrated as the orthotropic coordinate axis. The interface contains (111) and (11-1) facets. The sidewalls of the NWs denote any of the $\{111\}$ facets other than the (111) and (11-1) facets. The trijunction where vapor, liquid, and solid meet is labeled $t$. The contact angle $\theta_{c}$ is marked by two red lines, while dash lines represent the stable range of $\theta_{c}$. (b)-(i) are the specific process in the VLS growth (b)-(g) and in the VSS growth (h) and (i) of Ge NWs on Ge (110) substrate. The blue circles denote step forming in the process. We only choose the vertically grown NWs for example.

dominate, while their inclined counterparts are rare, i.e., the depinning process does not cross the left trijunction, remains unclear, but may originate from the low probability. During further NW growth, the depinned droplet slides along the (11-1) facet until forming a new step shown in Fig. 7(d). When the overall growth rate is low (which is the case in this study), the larger wetting facet has a higher growth velocity than that of the smaller one,$^{30}$ leading to a symmetric growth front shown in Fig. 7(e). The long growth time of several hours can cause a gradually decreasing the size of the droplet shown in Fig. 7(f), resulting in a mismatch between the catalyst size and the NW diameter. Then, the jumping-catalyst growth of alternating the droplet from one facet to another may occur, ${ }^{31,32}$ as indicated in Fig. 7(g). The growth process of the inclined NWs is identical to the above mechanism but rarely occurs.

In contrast, during the VSS growth, the phase of $\mathrm{Au}$ nanoparticles is solid, which has an abrupt interface with the NW tip. In this case, we assume that a catalytic nanoparticle may only sit on one $\{111\}$ facet of a pedestal, as shown in Fig. 7(h) with the tilted catalyst/NW interface satisfying the result in Fig. 4(b). Due to an abrupt solid/solid interface throughout the growth, the nanoparticle may tightly attach to the $\{111\}$ facet of the pedestal to advance one of the five equivalent $\langle 110\rangle$ growth directions, including a vertical one, two $30^{\circ}$-tilted directions and two lateral directions. Figure 7(i) exhibits one such situation as an example. Thus, the process during two-step growth has identical growth directions as seen in Fig. 3(a). As previously addressed in Figs. 2 and 4, the diameter of a NW equals to that of the solid Au nanoparticle in the VSS growth; however, it is larger than that of the Au catalyst in the VLS growth.

In accordance with the mechanism, the VSS growth has a significant impact on the NW size by efficiently decreasing the NW diameter and increasing its uniformity. Both advantages of the VSS growth on NWs morphology can result in a small diameter to enhance radial quantum confinement. In addition, during the VLS process, because Ge enters $\mathrm{Au}$ droplets at high temperatures, the catalyst becomes $\mathrm{Ge}$ supersaturated Au-Ge droplets. Contrarily, with the solid catalyst, the solubility of $\mathrm{Ge}$ in Au is lower than that in the VLS process. Solidified Au nanoparticles inhibit foreign Ge atoms injecting into themselves, ensuring the chemical purity of
NWs. ${ }^{13}$ This property determines that the average diameter of the catalysts in the VLS process remains larger than that in the VSS process, as shown in Fig. 4(a).

\section{CONCLUSION}

In summary, we have successfully grown Ge NWs on Ge (110) at $220^{\circ} \mathrm{C}$, the IC compatible temperature, by VSS $\mathrm{MBE}$, and found that the morphology both in growth direction and the dimension are different from these of the VLS process. Compared to the NWs under the VLS process at higher temperatures than $T_{\mathrm{e}}$, both the diameter and length decrease significantly in the VSS process. The diameters of Ge NWs under the VSS process strictly follow the size of Au nanoparticles, leading to a controllable growth. Simultaneously, pure NWs can be obtained from the VSS growth due to the prohibited mixing of Ge with the solid Au catalyst. The two-step growth of Ge NWs from the VLS growth switching to the VSS growth has been realized. Ge NWs grown under the second VSS process show the similarity in three growth orientations and the same diameter as that of the NWs directly grown at $220^{\circ} \mathrm{C}$.

\section{ACKNOWLEDGMENTS}

This study was supported by the Natural Science Foundation of China (61404153), the Shanghai Pujiang Program (14PJ1410600), the Key Research Program of the Chinese Academy of Sciences (KGZD-EW-804), and the Creative Research Group Project of Natural Science Foundation of China (61321492).

${ }^{1}$ M. Bosi and G. Attolini, Prog. Crystal Growth Charact. Mater. 56, 146 (2010).

${ }^{2}$ S.-J. Sun, P.-Y. Yang, S.-P. Ju, and Z.-M. Lai, J. Appl. Phys. 120, 195701 (2016).

${ }^{3}$ A. B. Greytak, L. J. Lauhon, M. S. Gudiksen, and C. M. Lieber, Appl. Phys. Lett. 84, 4176 (2004).

${ }^{4}$ G. Rosaz, B. Salem, N. Pauc, A. Potié, P. Gentile, and T. Baron, Appl. Phys. Lett. 99, 193107 (2011).

${ }^{5}$ M. M. Koleśnik-Gray, C. Sorger, S. Biswas, J. D. Holmes, H. B. Weber, and V. Krstić, Appl. Phys. Lett. 106, 233109 (2015).

${ }^{6}$ Y. Ahn and J. Park, Appl. Phys. Lett. 91, 162102 (2007).

${ }^{7}$ U. Otuonye, H. W. Kim, and W. D. Lu, Appl. Phys. Lett. 110, 173104 (2017).

${ }^{8}$ T. Kennedy, E. Mullane, H. Geaney, M. Osiak, C. O'Dwyer, and K. M. Ryan, Nano Lett. 14, 716 (2014).

${ }^{9}$ T. Hanrath and B. A. Korgel, J. Am. Chem. Soc. 124, 1424 (2002). 
${ }^{10} \mathrm{~J}$. Schmidtbauer, R. Bansen, R. Heimburger, T. Teubner, and T. Boeck, J. Cryst. Growth 406, 36 (2014).

${ }^{11}$ J. Schmidtbauer, R. Bansen, R. Heimburger, T. Teubner, T. Boeck, and R. Fornari, Appl. Phys. Lett. 101, 043105 (2012).

${ }^{12}$ J. Hannon, S. Kodambaka, F. Ross, and R. Tromp, Nature 440, 69 (2006).

${ }^{13}$ J. L. Lensch-Falk, E. R. Hemesath, D. E. Perea, and L. J. Lauhon, J. Mater. Chem. 19, 849 (2009).

${ }^{14}$ K. Kang, D. A. Kim, H. S. Lee, C. J. Kim, J. E. Yang, and M. H. Jo, Adv. Mater. 20, 4684 (2008).

${ }^{15}$ S. V. Thombare, A. F. Marshall, and P. C. McIntyre, APL Mater. 1, 061101 (2013).

${ }^{16}$ J. L. Lensch-Falk, E. R. Hemesath, and L. J. Lauhon, Nano Lett. 8, 2669 (2008).

${ }^{17}$ S. Kodambaka, J. Tersoff, M. Reuter, and F. Ross, Science 316, 729 (2007).

${ }^{18}$ M. Kolíbal, R. Kalousek, T. Vystavěl, L. Novák, and T. Šikola, Appl. Phys. Lett. 100, 203102 (2012).

${ }^{19}$ F. P. McCluskey, T. Podlesak, and R. Grzybowski, High Temperature Electronics (CRC Press, 1996).

${ }^{20}$ A. Y. Cho and J. Arthur, Prog. Solid State Chem. 10, 157 (1975).
${ }^{21}$ V. Dubrovskii, G. Cirlin, I. Soshnikov, A. Tonkikh, N. Sibirev, Y. B. Samsonenko, and V. Ustinov, Phys. Rev. B 71, 205325 (2005).

${ }^{22}$ H. Adhikari, A. F. Marshall, C. E. Chidsey, and P. C. McIntyre, Nano Lett. 6, 318 (2006).

${ }^{23}$ S. Biswas, J. Doherty, D. Saladukha, Q. Ramasse, D. Majumdar, M. Upmanyu, A. Singha, T. Ochalski, M. A. Morris, and J. D. Holmes, Nat. Commun. 7, 11405 (2016).

${ }^{24}$ M. Kolíbal, T. Pejchal, T. Vystavěl, and T. Šikola, Nano Lett. 16, 4880 (2016).

${ }^{25}$ D. McLachlan and E. G. Ehlers, J. Geophys. Res. 76, 2780, doi:10.1029/ JB076i011p02780 (1971).

${ }^{26}$ J. Johansson, C. P. T. Svensson, T. Mårtensson, L. Samuelson, and W. Seifert, J. Phys. Chem. B 109, 13567 (2005).

${ }^{27}$ V. Dubrovskii, N. Sibirev, G. Cirlin, J. Harmand, and V. Ustinov, Phys. Rev. E 73, 021603 (2006)

${ }^{28}$ P. W. Voorhees, J. Stat. Phys. 38, 231 (1985).

${ }^{29}$ K. Schwarz and J. Tersoff, Nano Lett. 11, 316 (2011).

${ }^{30}$ K. Schwarz and J. Tersoff, Phys. Rev. Lett. 102, 206101 (2009).

${ }^{31}$ K. Schwarz, J. Tersoff, S. Kodambaka, and F. Ross, Phys. Rev. Lett. 113, 055501 (2014).

${ }^{32}$ M. Kolíbal, T. Vystavěl, P. Varga, and T. Šikola, Nano Lett. 14, 1756 (2014). 